\title{
Spontaneous shape discrimination by frogs during unconditioned escape behavior
}

\author{
DAVID INGLE* \\ Neuropsychology Labrratory, McLean Hospital, Belmont, Massachusetts 02178
}

\begin{abstract}
Frogs were induced by visual threat to jump towards apertures in order to escape from a dark enclosure. These Ss consistently preferred to escape toward horizontal rather than toward vertical holes.
\end{abstract}

Although frogs are notoriously dependent upon visual motion in recognition of prey objects, they do attend to the precise distance and location of stationary objects when these are interposed as barriers (Ingle, 1970, 1971a). Furthermore, in an earlier study (Ingle, 1971b) where barriers to prey catching consisted of horizontal or vertical grids, frogs demonstrated a superior ability to avoid the vertical rather than the horizontal lines. This evidence suggests that some part of the frog's visual system is attuned to stationary edges of a specific orientation, as is known to be true of many neurons of the mammalian visual cortex. The question then arises as to whether frogs can spatially integrate such stationary-edge "detectors" in order to discriminate shapes of stationary objects. During pilot studies, it became evident that frogs pursued by threatening stimuli would orient to and leap through windows cut in surrounding enclosures, providing the aperture was large enough for the frog to negotiate. Since the profile of a jumping frog is horizontally elongated, teleological reasoning suggests that frogs might jump preferentially through horizontal apertures as opposed to those oriented vertically. The present study uses a two-hole forced-choice procedure to test this teleological hypothesis. Control tests were included to be certain that any observed preferences were not attributable to differential heights of the lower edge, which the frogs would have to clear during their jump.

\section{METHOD}

The Ss were 21/2-3-in.-long Rana pipiens, obtained from Mogul-Ed, Oskosh, Wisconsin, during the months of May and June. All frogs were kept within individual laboratory cages for a few weeks prior to the present study. During pilot tests, frogs were selected for their persistent tendency to avoid a threatening object passing about 6 in. over the head. Most of the frogs initially selected were used on most of the seven test series, and additional Ss were added as needed to replace those that became less responsive or that were used for other experiments. Each

*The author was supported by a National Institute of Mental Health Research Career Development Award (KO2 13175). Valuable technical assistance was provided by McLean Hospital volunteer staff members, Judith Grob and Fran Levitt. test series included 10 frogs, and the seven test series involved a total of 22 Ss.

As Fig. 1 shows, the test consisted simply of placing a frog at one corner of a $10 \times 10$ in. diamond-shaped enclosure, with the nose just entering the area, and inducing a vigorous escape response by bringing the E's hand directly to the top of the enclosure over the frog's head. A practiced motion bringing the hand along a 30-deg angle to a location 6 in. over the head invariably elicited a forward jump, if the frog had rested in place for at least $5 \mathrm{sec}$. Close observations indicated that frogs sitting slightly asymmetrically did not tend to jump toward the more frontal escape window, nor did asymmetrical hand placement induce a noticeable bias in response direction. As a matter of fact, in a separate study of directional avoidance patterns $\left(\right.$ Ingle $\left.^{1}\right)$, stimuli approaching the frog from 45 deg lateral to the rear midline did not induce asymmetrical jump directions. Therefore, this simple procedure seems suitable for testing the frog's discrimination of characteristics of the escape windows themselves.

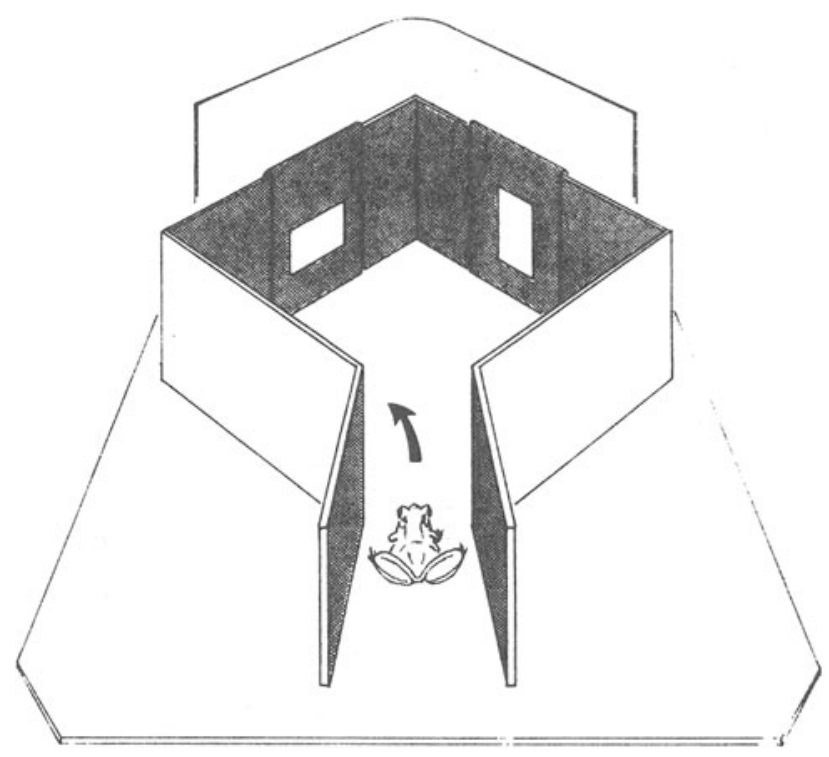

Fig. 1. Apparatus used for spontaneous discrimination of windows. When threatened from above, the frog jumps toward one of the two windows in an attempt to escape from the enclosure. A white background provides good contrast to the flat black inner walls. In this figure, the window centers are the same height, but in other tests, the lower edges were equated for height. The entire structure rests within one corner of a 3-ft-square walled arena. 


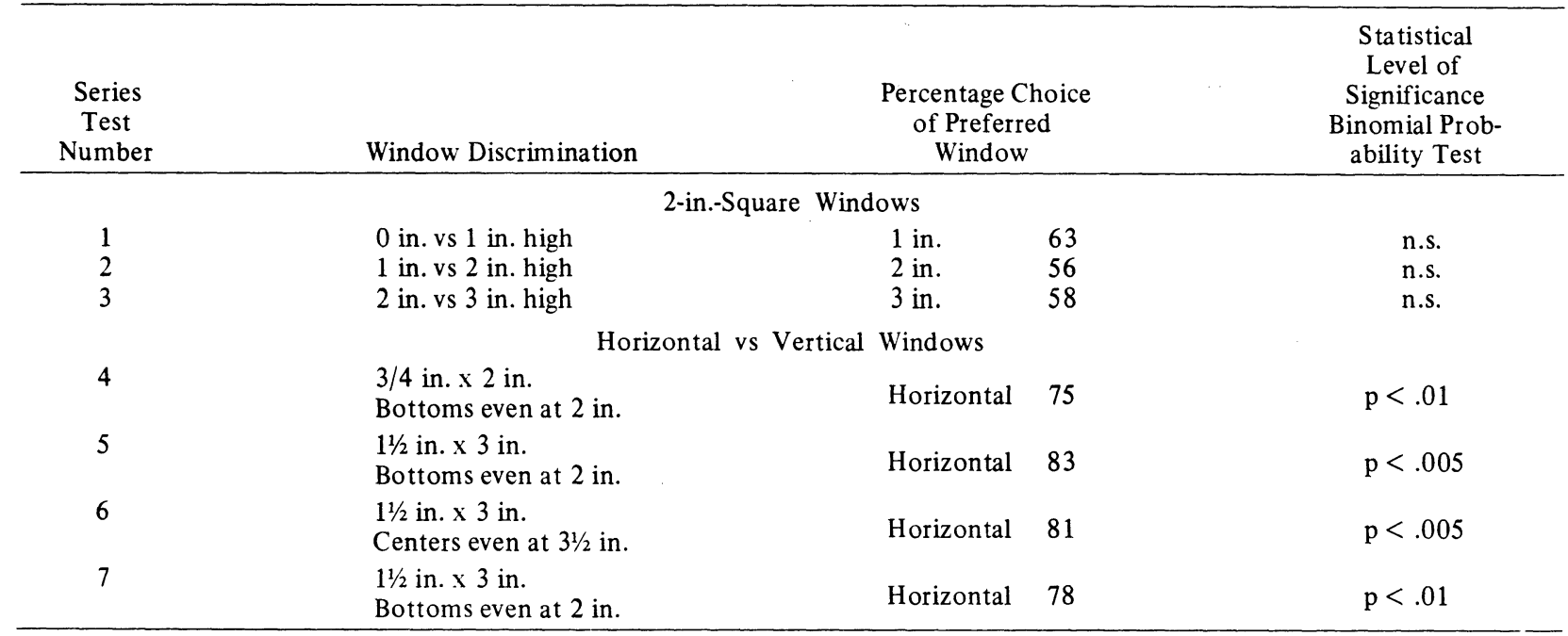

During each test session, frogs were tested two at a time on alternate trials in order to reduce any tendency to habituation to threat. Each session consisted of 10 trials per frog, in which Ss could escape through either of two windows cut in sheets of plastic that fitted onto either front wall of the enclosure, over a circular window cut in the enclosure itself (Fig. 1). On successive trials, these removable window pieces were alternated from left to right in a quasirandom sequence. Since the enclosure and the window pieces were painted flat black, a white plastic background was set 6 in. behind the window (against the outer wall of the 3 -ft-square test arena) to provide maximum contrast. In response to the descending hand, frogs jumped at least halfway toward one of the two windows, and never toward the center corner. Occasionally a frog jumped directly through one window via a single leap, but more frequently two hops were required to escape the enclosure. After an escape, frogs were allowed to rest for $5 \mathrm{sec}$ and were then gently nudged with a plastic barrier toward the nearest open region of the arena. In the event that a frog jumped from the starting position prematurely or turned in the cul-de-sac, the trial was reinitiated.

The first three test series were designed to determine whether the height of the escape hole from the floor induced consistent preferences. It seemed possible that a frog would jump no higher than necessary to escape, so that holes of different shapes might have to be equated for the absolute height of the lower edge. Using groups of 10 frogs, 100 choice responses were obtained for each of the following heights of pairs of 2-in. square holes: (1) 0 vs 1 in., (2) 1 vs 2 in., and (3) 2 vs 3 in., where the lower edge is measured from the floor. The remaining series consisted of horizontal vs vertical windows of the following dimensions: (4) $2 \times 3 / 4$ in., with bottom edges each 2 in. high, (5) $3 \times 1 \frac{1}{2}$ in., with bottom edges each 2 in. high, (6) $3 \times 1 \frac{1}{2}$ in., with the hole centers at the same $3 \frac{1}{1} 2$-in. height, and (7) a replication of Series 5.

\section{RESULTS}

Data from the first series of square hole tests, as illustrated in Table 1, indicate that the absolute height of top or bottom edges of the escape apertures has no statistically significant effect on jumping preference. However, a trend toward preference for the 1-in.-high aperture over the floor level hole is noticeable (63\%) and cannot be dismissed as insignificant on the basis of this small sample of Ss and trials. On the other hand, when height of the lower edge is equated, Ss do prefer to jump towards the horizontal hole, whether the $2 \times 3 / 4$ in. or the $3 \times 1 \frac{1}{2}$ in. size is used. Furthermore, the same level of preference $(81 \%)$ is found when horizontal and vertical apertures are equated for height of centers. Considering the larger holes alone, the $80 \%$ preference for the horizontal stimulus, found over 300 test trials in Series 5,6 , and 7 , is highly significant.

\section{DISCUSSION}

The data strongly confirm the teleological hypothesis that frogs jump preferentially toward horizontal escape windows when paired against vertical windows of the same area and brightness. Since preferences were not observed between square holes set at 1 vs 2 in. or 2 vs 3 in. above the floor, it seems that shape alone must account for this preference. These findings bear an uncertain relationship to the shape-discriminating abilities of mammals. For example, it cannot be said that frogs have an "abstract" recognition of vertical or horizontal rectangles, since the preferences may occur only with holes within a limited size range. The simplest hypothesis holds that the vertical hole is avoided simply because vertical edges are the more effective in inhibiting approach tendencies in frogs, as in earlier experiments with grid barriers (Ingle, 1971b). This interpretation of frog behavior is in line with an earlier conclusion (Ingle, 1971c) that the shape recognition in fish does not show the invariance during transformations of size and rotation attributed to mammalian vision, but instead is based upon the salience of simple features normally used by the organism to guide approach and avoidance tendencies toward natural objects. 


\section{REFERENCES}

Ingle, D. Visuomotor functions of the frog optic tectum. Brain, Behavior, \& Evolution, 1970, 3, 57-71.

Ingle, D. Prey-catching behavior of Anurans toward moving and stationary objects. Vision Research, 1971a, 3, 447-456.

Ingle, D. Discrimination of edge-orientation by frogs. Vision Research, 1971b, 11, 1365-1367.

Ingle, D. The experimental analysis of visual behavior. In W. S.
Hoar and D. J. Randall (Eds.), Fish physiology. Vol. 5. New York: Academic Press, 1971c. Pp. 59-77.

\section{NOTE}

1. Ingle, D. Tectai mediation of directed avoidance to visual threat in the frog. In preparation.

(Received for publication November 22, 1972; accepted December 19, 1972.) 